\title{
Neuromedin B modulates phosphate-induced vascular calcification
}

\author{
Hyun-Joo Park ${ }^{1,2,3}$, Mi-Kyoung Kim ${ }^{1,2}$, Yeon Kim ${ }^{1,2,3}$, Hyung Joon Kim ${ }^{1,2,3}$, Soo-Kyung Bae ${ }^{2,3,4} \mathcal{E}$ Moon-Kyoung Bae ${ }^{1,2,3, *}$ \\ ${ }^{1}$ Department of Oral Physiology, ${ }^{2}$ Periodontal Disease Signaling Network Research Center (MRC), ${ }^{3}$ Dental and Life Science Institute, \\ ${ }^{4}$ Department of Dental Pharmacology, School of Dentistry, Pusan National University, Yangsan 50612, Korea
}

\begin{abstract}
Vascular calcification is the heterotopic accumulation of calcium phosphate salts in the vascular tissue and is highly correlated with increased cardiovascular morbidity and mortality. In this study, we found that the expression of neuromedin B (NMB) and NMB receptor is upregulated in phosphate-induced calcification of vascular smooth muscle cells (VSMCs). Silencing of NMB or treatment with NMB receptor antagonist, PD168368, inhibited the phosphate-induced osteogenic differentiation of VSMCs by inhibiting Wnt/ $\beta$-catenin signaling and VSMC apoptosis. PD168368 also attenuated the arterial calcification in cultured aortic rings and in a rat model of chronic kidney disease. The results of this study suggest that NMB-NMB receptor axis may have potential therapeutic value in the diagnosis and treatment of vascular calcification. [BMB Reports 2021; 54(11): 569-574]
\end{abstract}

\section{INTRODUCTION}

Vascular calcification is characterized by ectopic deposition of calcium phosphate in the form of hydroxyapatite crystals in the vasculature (1). It has been commonly found in aging, atherosclerosis, diabetes mellitus and chronic renal disease, and leads to increased cardiovascular morbidity and mortality (2). VSMCs undergo biomineralization and transdifferentiate into osteoblast-like or chondrocyte-like cells (3). Several studies have suggest that many risk factors and specific drivers, such as elevated inorganic phosphate, oxidative stress, growth factors, and pro-inflammatory cytokines, are involved in the active regulation of calcification in VSMCs (4).

Originally isolated from amphibian skin, bombesin-like peptides consist of bombesin, ranatensin, and phyllolitorin subfamilies (5). Subsequently, two mammalian bombesin-like peptides,

*Corresponding author. Tel: +82-51-510-8239; Fax: +82-51-510-8238; E-mail: mkbae@pusan.ac.kr

https://doi.org/10.5483/BMBRep.2021.54.11.089

Received 8 July 2021, Revised 26 August 2021, Accepted 6 October 2021

Keywords: Bombesin-like peptides, Neuromedin B, Neuromedin B receptor, Vascular calcification, Vascular smooth muscle cells gastrin-releasing peptide (GRP) and neuromedin B (NMB), were identified (6). Through their respective cognate receptors, both of these are involved in several physiological and pathological functions, such as exocrine and endocrine secretions, stress responses, inflammation, and cancers (7). Bombesin-like peptides are important for bone remodeling, particularly for osteoclastogenesis and bone formation. GRP stimulates osteoclast differentiation through the GRP receptor signaling (8). NMB-NMB receptor signaling regulates the osteoclast formation by enhancing the proliferation and survival of cells of this lineage (9) and osteoblasts in the autocrine or paracrine manner (10). Accumulating evidence suggests that the process of bone remodeling is similar to that of vascular calcification (11). We have recently shown that GRP inhibition ameliorates the phosphate-induced vascular calcification in vitro and in vivo (12).

The present study was designed to determine the novel function of the NMB-NMB receptor axis in phosphate-induced vascular calcification and to identify the underlying molecular mechanism(s) in VSMCs, which may provide a novel potential therapeutic target for treating the diseases associated with vascular calcification.

\section{RESULTS}

\section{Upregulation of NMB and NMB receptor expression in phosphate-induced calcification of VSMCs}

We first verified the induction of calcification in VSMCs using inorganic phosphate (Pi). VSMCs were incubated with various concentrations of Pi-containing calcification medium and then stained with Alizarin red S (ARS) to determine the deposited calcium (13). As shown in Fig. 1A, calcium deposition increased dramatically at 2.6 and $3.5 \mathrm{mM} \mathrm{Pi}$, but no deposits were seen with normal growth medium. Calcium content and alkaline phosphatase (ALP) activity in VSMCs was significantly induced under calcifying conditions (Fig. 1B, C). Next, we measured the levels of expression of NMB and NMB receptors in VSMCs with Pi-induced calcification. As shown in Fig. 1D, the expression of $N M B$ and $N M B$ receptor mRNA was prominently increased in the calcified VSMCs. The levels of NMB and NMB receptor proteins were significantly elevated in the calcified VSMCs (Fig. 1E). In addition, the level of secreted NMB protein progressively increased, with increasing severity of VSMC calci- 
fication (Fig. 1F).

\section{Inhibition of NMB reversed osteogenic transdifferentiation during vascular calcification}

To examine the role of NMB in Pi-induced VSMC calcification, we used PD168368, a specific antagonist of the NMB receptor (14), and NMB siRNA. The knockdown efficiency of the NMB siRNA was evident with reduced levels of NMB mRNA and protein (Supplementary Fig. 1). As shown in Fig. 2A, when exposed to the calcifying medium, both NMB-silenced and PD168368-treated cells showed significantly less calcium deposition. Calcium content and ALP activity also suggested that NMB silencing as well as PD168368 treatment markedly inhibited the calcification in VSMCs (Fig. 2B, C). Next, we evaluated the effect of NMB inhibition on the Pi-induced osteogenic differentiation of VSMCs. As shown in Fig. 2D, increased expression of

(A)

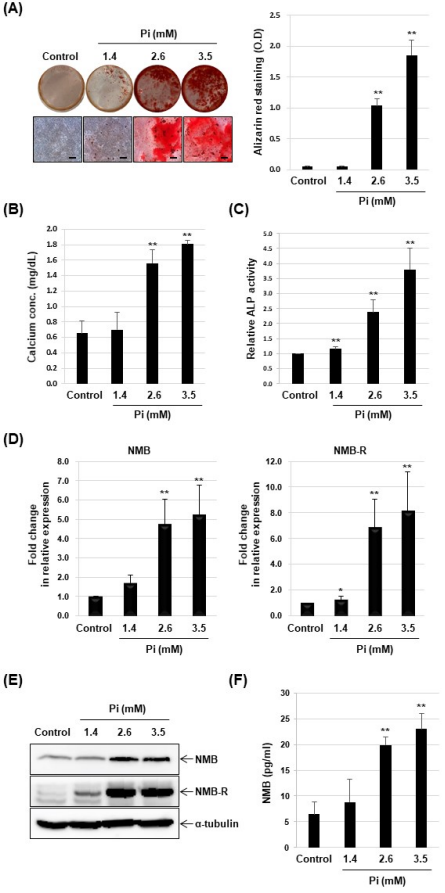

Fig. 1. Expression of $N M B$ and $N M B$ receptor in the phosphateinduced calcification of VSMC. VSMCs were cultured in growth medium or calcification medium for 9 days. (A) Calcified nodules were detected by ARS staining; nodules were photographed with a digital camera (upper) and observed using a phase contrast microscope (lower). Scale bar: $50 \mu \mathrm{m}$. The absorbance of the released ARS was measured to evaluate the degree of mineralization (right). (B and C) Total calcium content and ALP activity was measured. (D) Total RNA was isolated and analyzed using quantitative real-time RT-PCR with the specific primers for NMB and NMB-receptors. (E) The expression of $\mathrm{NMB}$ and $\mathrm{NMB}$ receptor protein was examined using western blotting. (F) The secreted NMB protein in the cell culture medium was measured using ELISA. ${ }^{*} \mathrm{P}<0.05 ; * * P<0.01$ vs. control.
Runx2 protein, a specific osteogenic marker, was inhibited by NMB silencing and PD168368 treatment. By contrast, the downregulation of calponin, a contractile phenotype maker, was completely reversed by NMB siRNA and PD168368. The immunofluorescence assay also showed that NMB silencing and PD168368 treatment lowered the level of Runx2 protein and enhanced that of calponin protein in calcified VSMCs (Fig. 2E). Furthermore, NMB silencing and PD168368 treatment upregulated the transcript level of calponin and downregulated those of Runx2 in VSMCs that have undergone Pi-induced osteogenic differentiation (Fig. 2F). Wnt/ $\beta$-catenin signaling is known to be involved in the phosphate-induced osteogenic differentiation of VSMCs (15). NMB silencing and PD168368 treatment abolished the induction of Wnt3a, dephosphorylated $\beta$-catenin

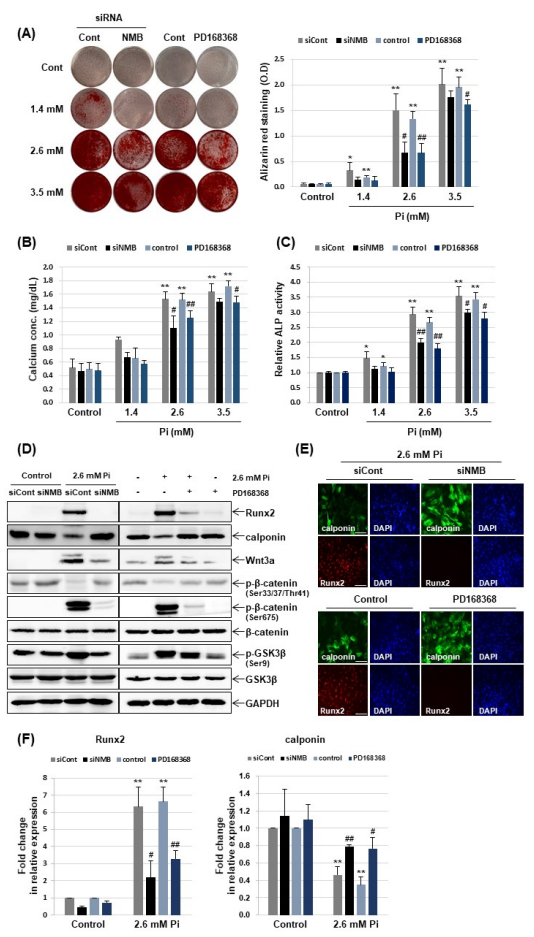

Fig. 2. Effect of NMB silencing and PD168368 treatment on the phosphate-induced phenotype switching of VSMCs. After transfection with siRNA for NMB or negative control, VSMCs were incubated in calcification medium. VSMCs were also cultured in calcification medium with or without PD168368 (100 nM). After 7 days, calcified nodules were detected by ARS staining and then absorbance was measured to evaluate the degree of mineralization (A). (B and $C$ ) Total calcium content and ALP activity was measured. (D) Changes in the levels of Runx2, calponin, Wnt3a, phospho- $\beta$-catenin (Ser33/37/

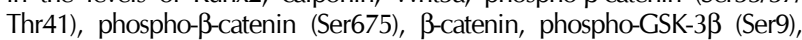
and GSK-3 $\beta$ proteins were evaluated using western blotting. (E) Immunocytochemical staining of Runx2 (red) and calponin (green) were visualized under a fluorescence microscope. Scale bar: $50 \mu \mathrm{m}$. (F) The mRNA levels of Runx2 and calponin were determined using quantitative real-time RT-PCR. ${ }^{*} \mathrm{P}<0.05 ; * * \mathrm{P}<0.01$ vs. control or control siRNA, ${ }^{\#} \mathrm{P}<0.05$; ${ }^{\# \#} \mathrm{P}<0.01$ vs. Pi. 


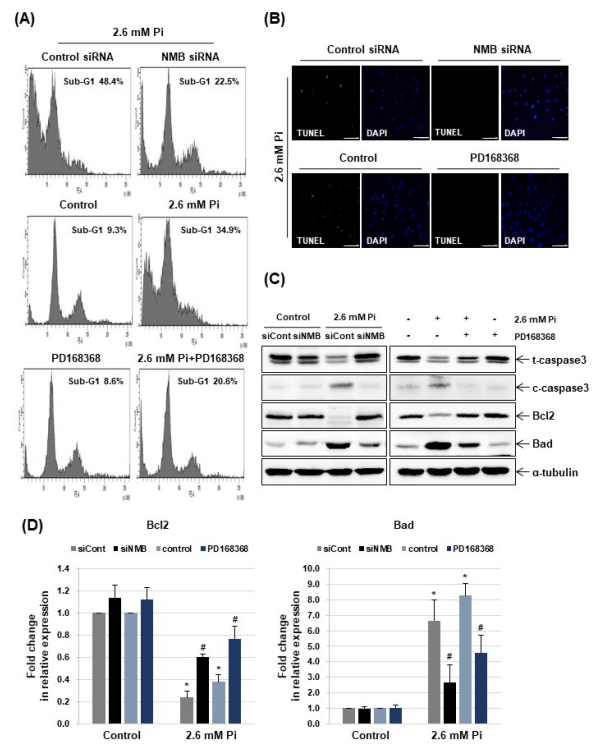

Fig. 3. Effect of NMB silencing and PD168368 treatment on phosphate-induced apoptosis of VSMCs. After transfection with siRNA for NMB or negative control, VSMCs were incubated with calcification medium with or without PD168368 (100 nM). (A) Apoptosis was detected using flow cytometry with PI staining. (B) The apoptotic bodies in VSMCs (green) were determined using TUNEL staining. DAPI (blue) stains nuclear DNA. Scale bar: $50 \mu \mathrm{m}$. (C) Changes in total/cleaved-caspase-3, Bcl2, and Bad were estimated using western blotting. (D) Using quantitative real-time RT-PCR, the expression levels of $\mathrm{BCl} 2$ and Bad mRNA were quantified. $* \mathrm{P}<0.01$ vs. control or control siRNA, ${ }^{\mathrm{P}}<0.01$ vs. $2.6 \mathrm{mM} \mathrm{Pi}$.

(Ser33/37/Thr41), and phosphorylated $\beta$-catenin (Ser675) by $2.6 \mathrm{mM} \mathrm{Pi}$ (Fig. 2D). In addition, given that the phosphorylation status of $\beta$-catenin can be achieved by glycogen synthase

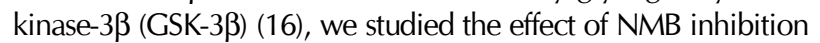
on GSK-3 $\beta$ in calcified VSMCs. As shown in Fig. 2D, both NMB siRNA and PD168368 blocked the Pi-induced phosphorylation of GSK-3 $\beta$ protein.

\section{Inhibition of NMB suppressed apoptosis in VSMC during vascular calcification}

Since apoptosis of VSMCs marks the initiation of vascular calcification (17), we assessed the apoptosis in VSMCs using propidium iodide staining. Compared to untreated cells, NMB-silenced and PD168368-treated VSMCs showed significantly less Pi-induced apoptosis (reduction of apoptotic cells by $25.9 \%$ and $14.3 \%$, respectively) (Fig. 3A). TUNEL-positive apoptotic cells also decreased markedly when VSMCs were treated with NMB siRNA or PD168368 (Fig. 3B). As shown in Fig. 3C, levels of cleavedcaspase- 3 and Bad proteins increased because of $2.6 \mathrm{mM} \mathrm{Pi}$ and decreased because of NMB silencing and PD168368 treatment. These two procedures also significantly induced the expression of $\mathrm{BCl} 2$ in calcified VSMCs (Fig. 3C). The excessive expression of Bad mRNA was inhibited by NMB silencing and

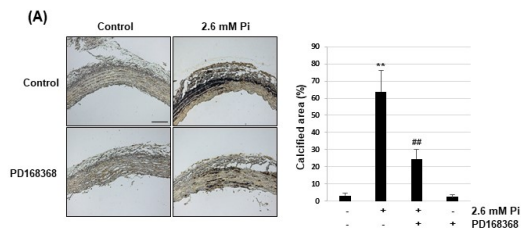

(B) (C)
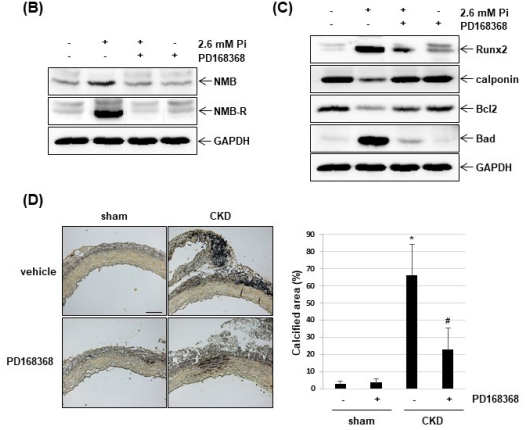

(E)

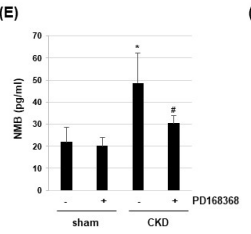

(F)

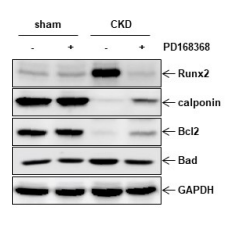

Fig. 4. Effect of PD168368 on the aortic calcification in ex vivo and in vivo. Pieces of rat aortas were cultured in calcification medium without or with $100 \mathrm{nM}$ PD168368. (A) The calcified lesions were stained with von Kossa staining. Scale bar: $50 \mu \mathrm{m}$ Percentage of the calcified area was calculated using calcification analyzer. (B and C) Expression of NMB, NMB receptor, Runx2, calponin, $\mathrm{BCl} 2$ and $\mathrm{Bad}$ protein were examined using western blotting. The CKD rats were fed a diet supplemented with adenine. For PD168368 treatment, CKD rats were injected intraperitoneally with PD168368 or vehicle. (D) Calcified lesions in aortas of sham and CKD rats were assessed using von Kossa staining. Scale bar: $50 \mu \mathrm{m}$. The percentage calcified area was calculated using calcification analyzer. (E) NMB protein levels were assessed using ELISA. (F) Western blots were individually probed with antibodies against Runx2, calponin, $\mathrm{BCl} 2$ and Bad. $* \mathrm{P}<0.05 ; * * \mathrm{P}<0.01$ vs. Control or sham, ${ }^{\#} \mathrm{P}<0.05 ;{ }^{\# \#} \mathrm{P} 0.01$ vs. $2.6 \mathrm{mM} \mathrm{Pi}$ or vehicle.

PD168368 treatment. By contrast, level of Bcl2 mRNA decreased in Pi-treated VSMCs compared to that in control, and this was completely reversed in the presence of $\mathrm{NMB}$ siRNA and PD 168368 (Fig. 3D).

\section{PD168368 attenuated the vascular calcification in ex vivo and in vivo}

To assess whether NMB regulates vascular calcification ex vivo (18), thoracic aortas were cultured in calcification medium with or without PD168368. As shown in Fig. 4A, under high phosphate conditions, vascular smooth muscle layers of the aorta showed extensive calcification, which was significantly reduced in those exposed to PD168368. Ratio of calcified area also decreased in the presence of PD168368. Furthermore, the protein expression of NMB and NMB receptor increased signifi- 
cantly in calcified aortic rings, but decreased drastically in presence of PD168368 (Fig. 4B). We also evaluated the effect of PD168368 on osteogenic differentiation and apoptosis in the calcified aortic rings. As shown in Fig. 4C, treatment with PD168368 abolished the Pi-induced reduction of calponin, as well as the enhancement of Runx2 to a great extent. Similarly, it reversed the reduction of $\mathrm{Bcl} 2$ and the promotion of Bad by Pi (Fig. 4C). Next, we adopted a rat model of chronic kidney disease (CKD) with vascular calcification (19) to demonstrate the role of NMB in vascular calcification in vivo. The blood biochemical parameters of the rat CKD model were summarized in S1 Table. As shown in Fig. 4D, the aortas of CKD rats had extensive calcification as compared with those from the sham rats. This was significantly alleviated in the aortas of the animals from PD168368-treated CKD group, resulting in reduced deposition of calcium as well as a small ratio of calcified area. The serum level of NMB was also increased in CKD rats compared with sham rats, which was restored to normal levels by PD 168368 treatment (Fig. 4E). Finally, western blotting demonstrated that PD168368 treatment blocked the osteogenic conversion and apoptosis in the aorta of CKD rats (Fig. 4F).

\section{DISCUSSION}

Mounting data suggest the critical role of mammalian bombesinlike peptides and their receptor signaling in the dysregulated vascular cell homeostasis. Both GRP and NMB enhance endothelial migration and angiogenic tube formation during neovascularization $(20,21)$. The GRP-GRP receptor is involved in the development and progression of atherosclerosis by stimulating the adhesion of monocytes to the activated vascular endothelium and migration and proliferation of VSMCs $(22,23)$. Calcification of the coronary arteries is commonly associated with the advanced stages of atherosclerosis (24). More work is needed to determine whether NMB can exert its effects on the vasculature, accelerating the stages of atherosclerosis, including endothelial dysfunction, initiation of atherosclerotic lesions, plaque progression, and destabilization and rupture of plaques.

Vascular calcification and bone disorder are often seen together in patients with CKD and increase the risk of cardiovascular mortality (25). Emerging clinical studies have demonstrated a relationship between the development of vascular calcification and dysregulation of bone mineral density in patients with CKD, promoting the concept of bone-vascular axis (26). The potential regulatory mechanisms and the shared molecular players involved in this axis have not yet been established. Bombesin-like peptides and their receptors may impact the bone homeostasis by modulating the differentiation, survival, and proliferation of the osteoclast lineage cells. GRP promotes osteoclastogenesis by stimulating the differentiation of osteoclast precursor cells into osteoclasts (8). Knockdown of NMB or NMB receptors suppresses the proliferation of osteoclast precursor cells and prevents the generation of osteoclasts (9). Wnt/ $\beta$-catenin signaling could play a crucial role in the regulation of high phosphate-induced calcification of VSMCs via the induction of osteogenic genes such as Runx2 (15). The present study and other reports $(27,28)$ demonstrated that high phosphate induces two active forms of $\beta$-catenin, $\beta$-catenin dephosphorylated at Ser37/Thr41 via the inactivation of GSK-3 $\beta$ through Ser9-phosphorylation and $\beta$-catenin phosphorylated at Ser675, resulting in promotion its stability and transcriptional activity. These changes were completely reversed by treatment with $\mathrm{NMB}$ siRNA or PD168368 (Fig. 2D), indicating that the NMB-NMB receptor axis could modulate high phosphate-induced VSMC calcification via $\mathrm{Wnt} 3 \mathrm{a} / \mathrm{\beta}$-catenin signaling pathway. In light of the present data, these observations suggest that bombesin-like peptides may serve as the common regulators of bone disorders and vascular calcification. In addition, it is necessary to address the specific mechanism by which bombesin-like peptides act in the bonevascular axis. We cannot exclude the possibility that the bombesin-like peptides are related to the bone regulatory proteins, such as RANK/RANK ligand/osteoprotegerin system (29) involved in the bone-vascular axis.

Plasma GRP levels increase significantly in the rat model of nephrectomy-induced uremia or adenine-induced CKD (12). Recently, serum proGRP levels have been reported to be considerably elevated in acute renal injury and in patients with CKD, and they increase with the severity of CKD (30). We demonstrated the elevated level of $\mathrm{NMB}$ in the serum of the CKD rat model. It is necessary to evaluate the expression of NMB at different stages of CKD and investigate whether they can be associated with the progression or severity of arterial calcification among CKD patients.

In the present study, we demonstrated that NMB-NMB receptor axis modulates the phosphate-induced calcification of VSMCs, and aortic calcification in organ culture of rat aorta ex vivo and in the aortas of rats with CKD in vivo. Our findings may help in understanding the potential role of NMB in the pathogenesis of vascular calcification and offer a promising therapeutic target for the prevention and treatment of diseases associated with vascular calcification.

\section{MATERIALS AND METHODS}

All animal studies were conducted in accordance with the Guide for the Care and Use of Laboratory Animals (NIH publication No. 85-23, revised 1996) and were approved by the Institutional Animal Care and Use Committee at Pusan National University, Korea.

\section{Reagents and antibodies}

PD168368 was purchased from Tocris Bioscience. The antibodies against NMB and NMB receptor were supplied by Thermo Fisher Scientific and Santa Cruz Biotechnology, respectively. Antibodies against phospho- $\beta$-catenin (Ser33/37/Thr41), phospho$\beta$-catenin (Ser675), $\beta$-catenin, phospho-GSK-3 $\beta$ (Ser9), GSK-3 $\beta$, Runx2, total/cleaved-caspase-3, Bcl2, and Bad were procured from Cell Signaling Technology. Wnt3a and calponin antibodies 
were purchased from Abcam and anti $\beta$-actin antibody was from Bioworld Technology.

\section{Cell isolation and culture}

VSMCs isolated from thoracic aortas of male Sprague-Dawley rats (3-week-old, Samtaco) were cultured in Dulbecco's modified Eagle's medium (DMEM) with 10\% fetal bovine serum and 1\% antibiotics (all from Thermo Fisher Scientific) at $37^{\circ} \mathrm{C}$ in a humidified $95 \%$ air and $5 \% \mathrm{CO}_{2}$ environment.

\section{Induction and quantification of calcification}

A solution of inorganic phosphate $(\mathrm{Pi})\left(\mathrm{Na}_{2} \mathrm{HPO}_{4}\right.$ and $\mathrm{NaH}_{2} \mathrm{PO}_{4}$, $\mathrm{pH}$ 7.4) was added to the serum supplemented-DMEM at the final concentrations of $1.4,2.6$, and $3.5 \mathrm{mM}$. After the indicated incubation period, cellular calcium content and ALP activity were determined using calcium colorimetric assay kit (BioVision) and ALP assay kit (Takara), respectively.

\section{ARS staining}

Cells grown on plastic supports were fixed with $4 \%$ paraformaldehyde and stained with $1 \mathrm{mg} / \mathrm{ml}$ ARS (Sigma) solution for $30 \mathrm{~min}$ at $37^{\circ} \mathrm{C}$. Samples were rinsed, and the stained calcium deposits were photographed. Once the images were acquired, calcium deposits were destained and dissolved in $10 \%$ acetic acid. The absorbance at $420 \mathrm{~nm}$ was measured using a multidetection microplate reader (Dynex) to quantify the calcification.

\section{Von Kossa staining}

After dewaxing in xylene and rehydrating through a graded series of alcohol, slides carrying tissue slices were soaked in distilled water and incubated in $1 \%$ silver nitrate solution (Sigma) under a UV lamp for $15 \mathrm{~min}$. They were subsequently soaked in $5 \%$ sodium thiosulfate for $5 \mathrm{~min}$ and cleaned with distilled water; then, the sections were incubated with nuclear fast red for $5 \mathrm{~min}$ at $23-25^{\circ} \mathrm{C}$ and dehydrated using alcohol grade series.

\section{Enzyme-linked immunosorbent assay (ELISA)}

The amount of secreted NMB protein was determined using ELISA following the manufacturer's instructions (MyBioSource). The absorbance of the samples at $450 \mathrm{~nm}$ was measured using an ELISA reader (Dynex), and the NMB levels were determined by interpolating the values on to a standard curve generated according to the manufacturer's instructions.

\section{Gene knockdown by small-interfering RNA (siRNA)}

The small interfering (si) RNA duplexes for rat NMB and a negative control siRNA were bought from GenePharma. VSMCs were transfected using Amaxa Nucleofector (Lonza) according to the manufacturer's instructions.

\section{Flow cytometry analysis}

VSMCs were incubated in calcification medium for 7 days, washed twice in 1X PBS, and fixed in chilled $70 \%$ ethanol. The cells were stained with $5 \mu \mathrm{g} / \mathrm{ml}$ propidium iodide (Sigma) at $23-25^{\circ} \mathrm{C}$ for $10 \mathrm{~min}$ and analyzed using FACS Calibur (BD Bioscience). The cell cycle profile was determined using the Modfit LT software.

\section{TUNEL assay}

Apoptotic cells were detected using the DeadEnd ${ }^{\mathrm{TM}}$ Fluorometric TUNEL System (Promega) in accordance with the manufacturer's instructions. Cells were incubated in calcification medium for 7 days, fixed in $4 \%$ paraformaldehyde for $25 \mathrm{~min}$ at $4{ }^{\circ} \mathrm{C}$, and permeabilized with $0.2 \%$ Triton $\mathrm{X}-100$ for $5 \mathrm{~min}$ at room temperature. Free $3^{\prime}$-ends of the fragmented DNA were labeled in the TUNEL reaction mixture for $60 \mathrm{~min}$ at $37^{\circ} \mathrm{C}$ in a humidified chamber and visualized under a fluorescence microscope (Nikon)

\section{Arterial ring calcification}

The thoracic aortas were excised in a sterile manner from male Sprague-Dawley rats (6-week-old, Samtaco). After removing the adventitia and endothelium, the vessels were cut into 2-3-mm rings and placed in either calcification medium or normal culture medium at $37^{\circ} \mathrm{C}$ in $5 \% \mathrm{CO}_{2}$ for 10 days, with medium change once every 3 days.

\section{Induction of CKD rat mode}

CKD was induced as described previously (31). Twelve-week-old male Wistar rats (Orientbio) were randomly divided into four groups: sham, CKD, sham + PD168368, CKD + PD168368. Animals from the sham groups were fed a normal diet, whereas those from the CKD groups were fed a diet supplemented with $0.75 \%$ adenine for 8 weeks. For PD168368 treatment, CKD mice were injected intraperitoneally with PD168368 (1.2 $\mathrm{mg} / \mathrm{kg} /$ day) or vehicle (PEG 400, Sigma) three times a week for 8 weeks.

\section{Quantitative real-time RT-PCR, western blot analysis, and immunocytochemistry}

Quantitative real-time RT-PCR, western blot analysis, and immunocytochemistry were performed as described in the Supplementary material.

\section{Statistical analyses}

Data are the mean \pm standard deviation of at least three independent experiments. Statistical comparisons between groups were performed by the one-way analysis of variance followed by the Student t-test.

\section{ACKNOWLEDGEMENTS}

This research was supported by the National Research Foundation of Korea grant, funded by the Korean government (MSIT) (NRF2018R1A5A2023879) (to M-K Bae), and Basic Science Research Program through the National Research Foundation of Korea funded by the Ministry of Education (NRF-2019R1I1A1A01061 377) (to H-J Park). 


\section{CONFLICTS OF INTEREST}

The authors have no conflicting interests.

\section{REFERENCES}

1. Johnson RC, Leopold JA, and Loscalzo J (2006) Vascular calcification: pathobiological mechanisms and clinical implications. Circ Res 99, 1044-1059

2. Singh A, Tandon S, and Tandon C (2021) An update on vascular calcification and potential therapeutics. Mol Biol Rep 48, 887-896

3. Leopold JA (2015) Vascular calcification: mechanisms of vascular smooth muscle cell calcification. Trends Cardiovasc Med 25, 267-274

4. McCarty MF and DiNicolantonio JJ (2014) The molecular biology and pathophysiology of vascular calcification. Postgrad Med 126, 54-64

5. Jensen RT, Battey JF, Spindel ER, and Benya RV (2008) International union of pharmacology. LXVIII. Mammalian bombesin receptors: nomenclature, distribution, pharmacology, signaling, and functions in normal and disease states. Pharmacol Rev 60, 1-42

6. Majumdar ID and Weber HC (2011) Biology of mammalian bombesin-like peptides and their receptors. Curr Opin Endocrinol Diabetes Obes 18, 68-74

7. Gonzalez N, Moody TW, Igarashi $\mathrm{H}$, Ito $\mathrm{T}$, and Jensen RT (2008) Bombesin-related peptides and their receptors: recent advances in their role in physiology and disease states. Curr Opin Endocrinol Diabetes Obes 15, 58-64

8. Choi Y, Heo SC, Kim YN et al (2020) Gastrin-releasing peptide (GRP) stimulates osteoclastogenesis in periodontitis. Cells 10, 50

9. Yeo CE, Kang WY, Seong SJ et al (2017) Neuromedin B and its receptor silencing suppresses osteoclast generation by modulating precursor proliferation via M-CSF/c-Fms/D-type cyclins. Exp Cell Res 359, 112-119

10. Saito $H$, Nakamachi T, Inoue K et al (2013) Autocrine effects of neuromedin B stimulate the proliferation of rat primary osteoblasts. J Endocrinol 217, 141-150

11. Cannata-Andia JB, Roman-Garcia P, and Hruska K (2011) The connections between vascular calcification and bone health. Nephrol Dial Transplant 26, 3429-3436

12. Park HJ, Kim Y, Kim MK et al (2020) Inhibition of gastrinreleasing peptide attenuates phosphate-induced vascular calcification. Cells 9, 737

13. Jono S, McKee MD, Murry CE et al (2000) Phosphate regulation of vascular smooth muscle cell calcification. Circ Res 87, E10-7

14. Gonzalez N, Mantey SA, Pradhan TK et al (2009) Characterization of putative GRP- and NMB-receptor antagonist's interaction with human receptors. Peptides 30, 1473-1486

15. Cai T, Sun D, Duan Y et al (2016) WNT/beta-catenin signaling promotes VSMCs to osteogenic transdifferentiation and calcification through directly modulating Runx2 gene expression. Exp Cell Res 345, 206-217

16. Wu D and Pan W (2010) GSK3: a multifaceted kinase in Wnt signaling. Trends Biochem Sci 35, 161-168
17. Proudfoot D, Skepper JN, Hegyi L, Bennett MR, Shanahan CM, and Weissberg PL (2000) Apoptosis regulates human vascular calcification in vitro: evidence for initiation of vascular calcification by apoptotic bodies. Circ Res 87, 1055-1062

18. Akiyoshi $\mathrm{T}$, Ota $\mathrm{H}$, lijima $\mathrm{K}$ et al (2016) A novel organ culture model of aorta for vascular calcification. Atherosclerosis 244, 51-58

19. Shobeiri N, Adams MA, and Holden RM (2010) Vascular calcification in animal models of CKD: A review. Am J Nephrol 31, 471-481

20. Park HJ, Kim SR, Bae SK et al (2009) Neuromedin B induces angiogenesis via activation of ERK and Akt in endothelial cells. Exp Cell Res 315, 3359-3369

21. Martinez A, Zudaire E, Julian M, Moody TW, and Cuttitta F (2005) Gastrin-releasing peptide (GRP) induces angiogenesis and the specific GRP blocker 77427 inhibits tumor growth in vitro and in vivo. Oncogene 24, 4106-4113

22. Park HJ, Kim MK, Kim Y et al (2017) Gastrin-releasing peptide promotes the migration of vascular smooth muscle cells through upregulation of matrix metalloproteinase-2 and -9. BMB Rep 50, 628-633

23. Kim MK, Park HJ, Kim Y, Kim HJ, Bae SK, and Bae MK (2017) Gastrin-releasing peptide induces monocyte adhesion to vascular endothelium by upregulating endothelial adhesion molecules. Biochem Biophys Res Commun 485, 542-549

24. Rodriguez-Granillo GA, Carrascosa P, and Bruining N (2016) Progression of coronary artery calcification at the crossroads: sign of progression or stabilization of coronary atherosclerosis? Cardiovasc Diagn Ther 6, 250-258

25. Schlieper G, Schurgers L, Brandenburg V, Reutelingsperger C, and Floege J (2016) Vascular calcification in chronic kidney disease: an update. Nephrol Dial Transplant 31, 31-39

26. Evenepoel P, Opdebeeck B, David K, and D'Haese PC (2019) Bone-vascular axis in chronic kidney disease. Adv Chronic Kidney Dis 26, 472-483

27. Cong J, Cheng B, Liu J, and He P (2021) RTEF-1 inhibits vascular smooth muscle cell calcification through regulating Wnt/beta-Catenin signaling pathway. Calcif Tissue Int $109,203-214$

28. Song WJ, Song EA, Jung MS et al (2015) Phosphorylation and inactivation of glycogen synthase kinase 3beta (GSK3beta) by dual-specificity tyrosine phosphorylation-regulated kinase 1A (Dyrk1A). J Biol Chem 290, 2321-2333

29. Znorko B, Oksztulska-Kolanek E, Michalowska M, Kaminski T, and Pawlak K (2017) Does the OPG/RANKL system contribute to the bone-vascular axis in chronic kidney disease? A systematic review. Adv Med Sci 62, 52-64

30. Dai Z, Zhu J, Huang $\mathrm{H}$ et al (2020) Expression and clinical value of gastrin-releasing peptide precursor in nephropathy and chronic kidney disease. Nephrology (Carlton) 25, 398405

31. Tamagaki K, Yuan Q, Ohkawa $\mathrm{H}$ et al (2006) Severe hyper-parathyroidism with bone abnormalities and metastatic calcification in rats with adenine-induced uraemia. Nephrol Dial Transplant 21, 651-659 